\title{
A systematic review of factors associated with service user satisfaction with psychiatric inpatient services
}

DOI:

10.1016/j.jpsychires.2017.03.020

\section{Document Version}

Accepted author manuscript

Link to publication record in Manchester Research Explorer

\section{Citation for published version (APA):}

Woodward, S., Berry, K., \& Bucci, S. (2017). A systematic review of factors associated with service user satisfaction with psychiatric inpatient services. Journal of Psychiatric Research, 92, 81-93.

https://doi.org/10.1016/j.jpsychires.2017.03.020

\section{Published in:}

Journal of Psychiatric Research

\section{Citing this paper}

Please note that where the full-text provided on Manchester Research Explorer is the Author Accepted Manuscript or Proof version this may differ from the final Published version. If citing, it is advised that you check and use the publisher's definitive version.

\section{General rights}

Copyright and moral rights for the publications made accessible in the Research Explorer are retained by the authors and/or other copyright owners and it is a condition of accessing publications that users recognise and abide by the legal requirements associated with these rights.

\section{Takedown policy}

If you believe that this document breaches copyright please refer to the University of Manchester's Takedown Procedures [http://man.ac.uk/04Y6Bo] or contact uml.scholarlycommunications@manchester.ac.uk providing relevant details, so we can investigate your claim.

\section{OPEN ACCESS}




\section{A systematic review of factors associated with service user satisfaction with psychiatric inpatient services}

\section{Sarah Woodwarc}

Katherine Berry

Sandra Bucci*

Sandra.Bucci@manchester.ac.uk

Division of Psychology and Mental Health, School of Health Sciences, The University of Manchester, United States

${ }^{*}$ Corresponding author. Division of Psychology and Mental Health, Zochonis Building, University of Manchester, Brunswick Street, Manchester M13 9PL, United States

\section{Abstract}

\section{Background}

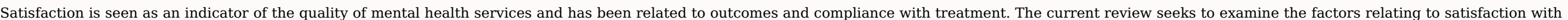
inpatient services.

\section{Method}

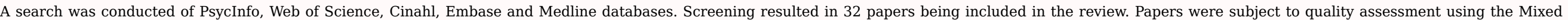
Methods Appraisal Tool (MMAT).

Results

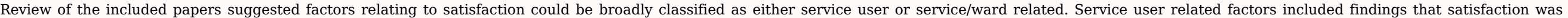

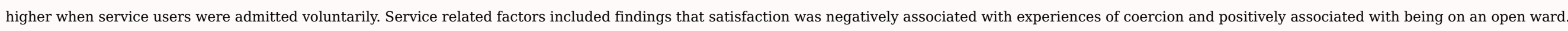
Conclusion

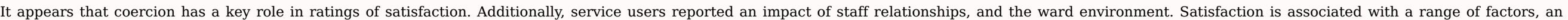
awareness of which will allow for the development of quality services that meet the needs of service users.

\section{Introduction}

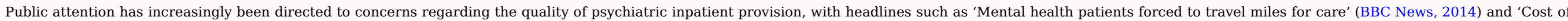

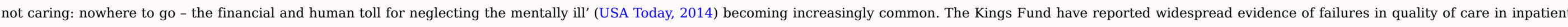

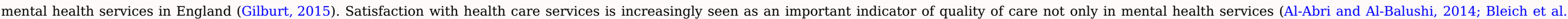

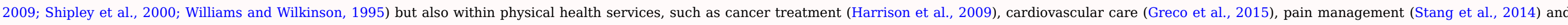

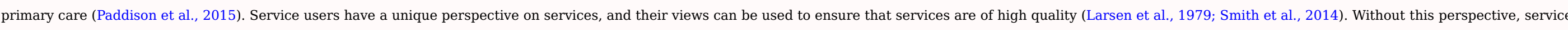

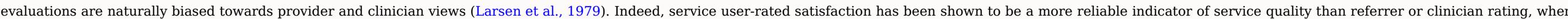

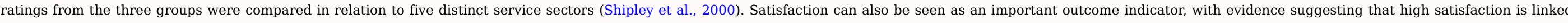

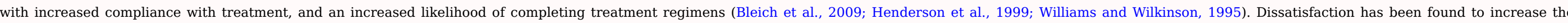

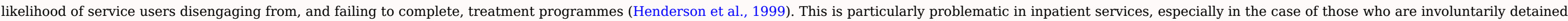

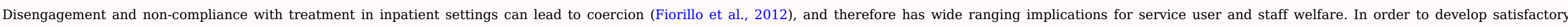
services, it is necessary to understand the factors that affect service users' satisfaction with services. 


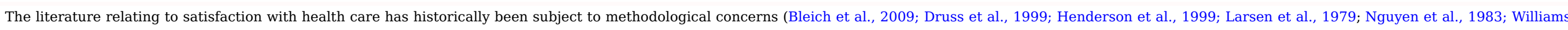

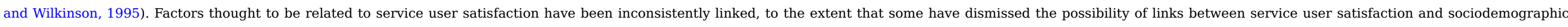

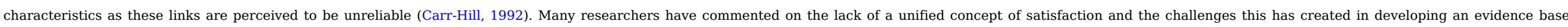

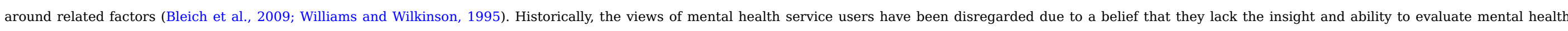

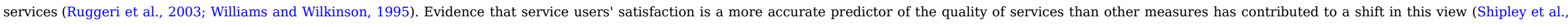

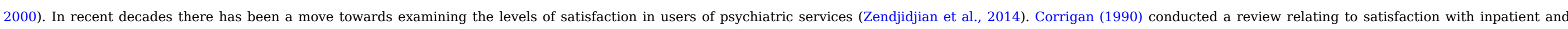

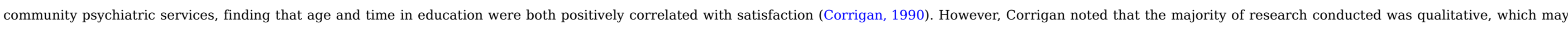

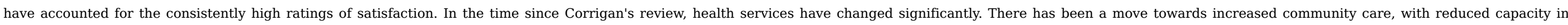
inpatient services. It is likely that these changes have impacted on the experiences of service users.

\subsection{Aims of the study}

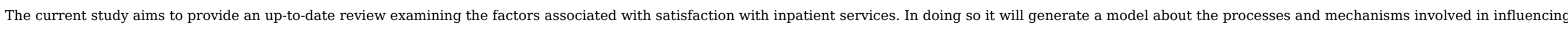

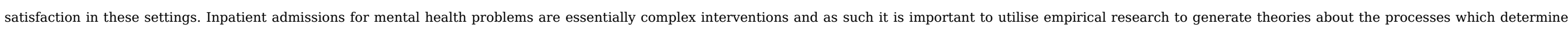
how these interventions work with a view to maximising future effectiveness in terms of patient outcomes including patient satisfaction (Medical Research Council, 2000, 2008).

\section{Method}

\subsection{Eligibility criteria}

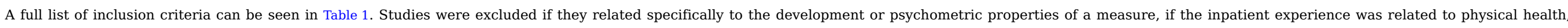
learning disability, child and adolescent, or older adult services, in order to ensure a homogenous sample, and if the views of inpatients were inseparable from those of another group (e.g. staff, carers).

Table 1 Inclusion criteria.

alt-text: Table 1

Inclusion criteria

Sample of former or current inpatients

Include an exploration of service user views of the inpatient environment

Published in a peer reviewed journal

English Language

Published post-1990

\subsection{Search strategy}

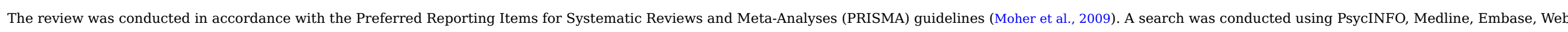

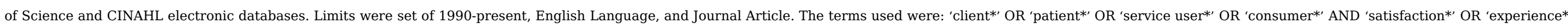
OR 'perception*' OR 'view*' OR 'opinion*' OR 'attitude*' OR 'evaluation*' AND 'severe mental*' OR 'schizophren*' OR 'psycho*' OR 'mental health*' AND 'inpatient*' OR 'ward*' OR 'psychiatr* serv*'.

\subsection{Study selection and data extraction}

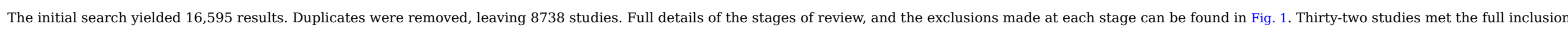

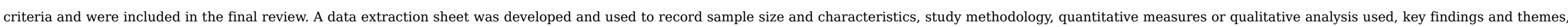




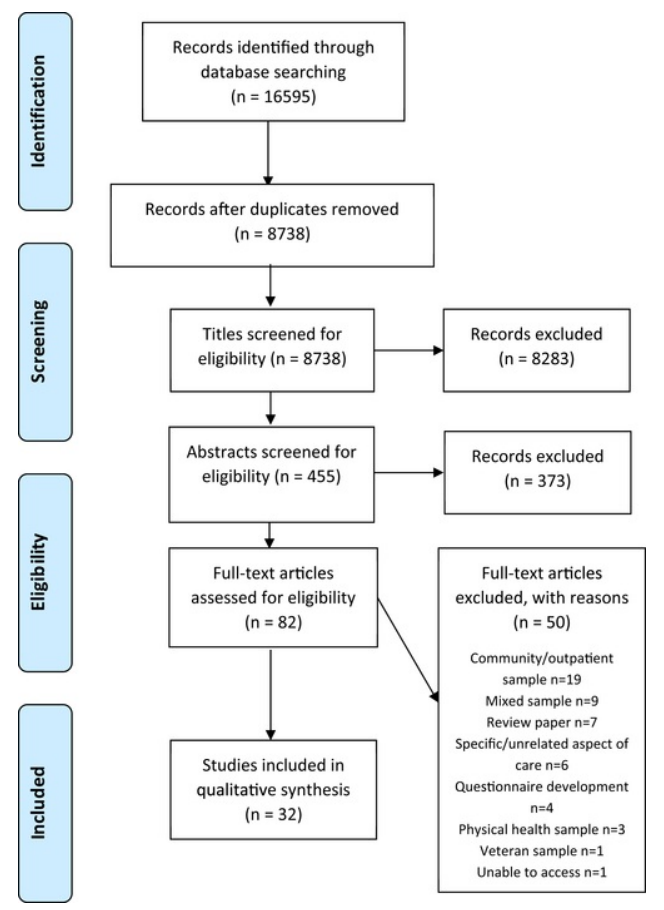

Fig. 1 PRISMA flow diagram.

\section{alt-text: Fig. 1}

\subsection{Quality assessment}

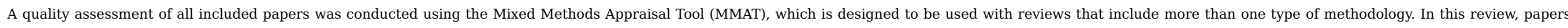

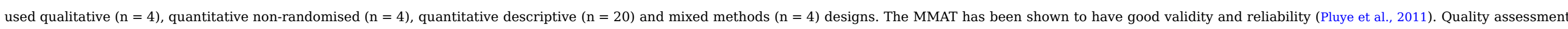

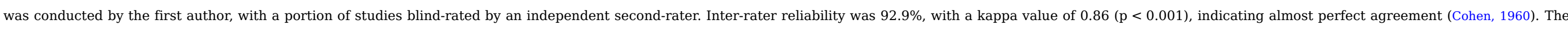

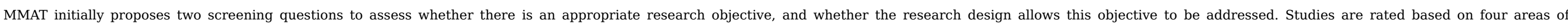
methodology, which vary depending on study methodology. Papers were assessed as either meeting the criteria, or not, and each area represents $25 \%$ of the total quality score (ranging from $0 \%$ to $100 \%$ ).

\section{Results}

\subsection{Overview of studies}

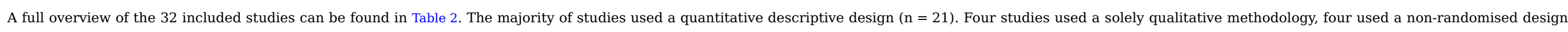

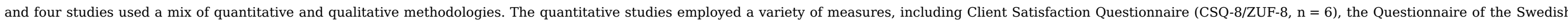

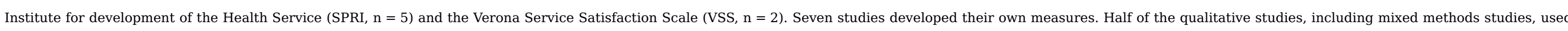

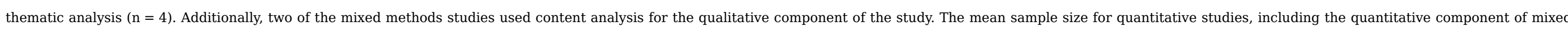

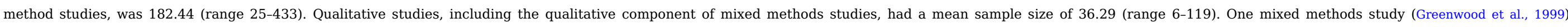

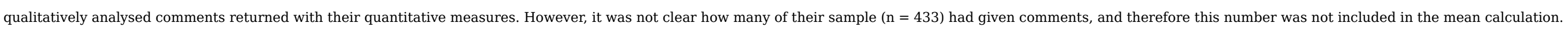


Table 2 Summary of findings.

alt-text: Table 2

Paper
-text: Table 2
characteristic

Setting

Measures/Analysis

Quality

Qsessment

score

\section{Alexius et \\ $\mathrm{N}=63$ former}

al. (2000)

inpatients, 3

months after their

stay

\section{Barker et \\ $\mathrm{N}=137$ current}

al. (1996)

inpatients

\section{Berghofer}

et al.

(2001)

$\mathrm{N}=165$ current

inpatients $(\mathrm{N}=72$

first time

inpatients, $\mathrm{N}=93$

long-term

inpatients.)

Boydell et $\mathrm{N}=216$ current

al. (2012) inpatients with first episode psychosis

Brunero et $\mathrm{N}=70$ inpatients

al. (2009) on day of discharg

un

Three

psychiatric

wards.

England

Acute inpatien

mental health

nit.

Australia

Cleary et

al. (2009)

$\mathrm{N}=100$ inpatients admitted to a new facility

Adult mental health facility Australia

Descriptive Inpatient Evaluation \begin{tabular}{l|l} 
cross- & of Services
\end{tabular} sectional Questionnaire (Meehan et al. 2002)

\section{Duggins}

(2006)

users with a

diagnosis of

schizophrenia,

discharged from

inpatient services

within the last year

Gebhardt $\quad \mathrm{N}=113$ inpatients

et al.

at the point of

discharge Psychiatry,

Psychotherapy cross-

\section{Descriptive SPRI (Hansson and} Hoglund, 1995)

Highest satisfaction

No effect of age or gender

Bespoke

questionnaire based

on previous

research, and sta opinions. Piloted.

Descriptive Developed a

questionnaire,

with staff,

environment and

other patients

Descriptive Acute Services

Study Questionnaire

(Sammut and Leff, 1995)

Descriptive Developed a

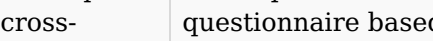

on national

standards, and

relevant literature.

$61.2 \%$ described themselves as satisfied with their care. Older patients were significantly more satisfied with \% psychosis $\left(\chi^{2}=12.24, p=0.02\right)$, those who were involuntarily admitted $\left(\chi^{2}=12.18, p=0.02\right)$, and those who felt they did not need to be in hospital (tau $=0.21, p=0.0003$ ). There was no effect of gender.

Overall positive satisfaction scores - 60\% first time, $68 \%$ long term. Most satisfied with staff, lest satisfied with other service users.

Mean number of items rated as satisfactory - 13 of 21. No difference between Black African and White British terms of total mean satisfaction. Black Caribbean service users significantly less satisfied than White service users $(t=2.56, p=0.006)$. Total satisfaction increased with age. No effect of gender, most social class groups, diagnosis of depression or compulsory treatment.

Overall satisfaction score mean of 6.8 out of 10 . Presence of community case manager significantly associated $25 \%$ with higher overall satisfaction ( $f=4.507, p=0.037$ ). Three questionnaire items significantly predicted satisfaction - happy with service provided by consumer support workers $(\beta=0.96), p<0.001)$, confidence in level of support following discharge $(\beta=0.64, p=0.007)$, feeling safe and secure of the ward $(\beta=0.53$,

$\mathrm{p}=0.022$ ). These items accounted for $50 \%$ of the variation in the overall satisfaction scale (adjusted

$\left.\mathrm{r}^{2}=0.51\right)$. Ward cleanliness was highly rated overall $(3.8 / 5)$, but was significantly lower in those who were in their first admission $\left(\chi^{2}=10.05, \mathrm{p}=0.04\right)$

$44 \%$ of patients rated their overall stay as better than expected. Overall satisfaction mean score was 3.13/5 No significant effects of gender or number of previous admissions.
Department of

Descriptive

Acute inpatient Qualitative Semi-structured

interviews,

cognitive mapping

analysis

Satisfaction was influenced by internal and external factors. External: fear of violence reduced satisfaction communication with staff increased satisfaction. Dissatisfaction with ward reviews, queueing for meals, ward activities and mixed sex wards. Internal: decreased satisfaction when staff were perceived to have a different understanding of their illness. Low expectations of services tended to increase satisfaction

Client Satisfaction

Satisfaction was negatively correlated with clinical well-being (CGI part $1 \mathrm{r}=-0.197, \mathrm{p}=0.036$, part 2 $r=-0.325, p<0.001)$ and positively correlated with global functioning $(r=0.239, p=0.011)$ at discharge. Medication side effects were associated with reduced satisfaction $(t=-3.02, p=0.003)$. There was $n$ significant effect of diagnosis, age, gender, number of admissions, duration of treatment and level of 
et al.

$\mathrm{N}=169$ current

(2002)

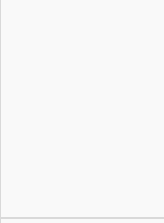

Gjerden

(1997)

$\mathrm{N}=135$ previously discharged inpatients

Greenwood $\quad \mathrm{N}=433$ current et al.

(1999)

inpatients

\begin{tabular}{l|l|l} 
Six acute & $\begin{array}{l}\text { Mixed } \\
\text { psychiatric }\end{array}$ & $\begin{array}{l}\text { Single genera } \\
\text { methods }\end{array}$ \\
satisfaction
\end{tabular}

wards.

England

Two hospital

sites.

$\mathrm{N}=215$ current

inpatients

Howard

Psychiatric ward, Genera

taly

Open ward,

central hospita

Norway

Descriptive

Descriptive

ectional

and an open

questionnaire

shown to have

satisfactory

psychometric

properties

SPRI

satisfaction

questionnaire -

likert scale.

CSQ-8.

analysis

cross- Satisfaction
Gigantesco N $=169$ current literature review

survey - tested and

Semi-structured

interview - content

Descriptive Kentucky Consumer

Instrument (Hov

Instrument (Hov

MHSIP-21 consume

survey (Jerrell,

2006)

CSQ-8

Two psychiatric Descriptive Questionnaire

wards at a

General

sectional

and other

questionnaires

Denmark

inpatients with

inpatients with

schizophrenia

$\mathrm{N}=217$ inpatients

with unipolar

depression), at

point of either

admission or

discharge

(2006) inpatients
Psychiatric unit. Naturalistic Zurich Satisfaction Germany trial

\section{Questionnaire (ZUF-}

Kohler et $\quad \mathrm{N}=292(\mathrm{~N}=75$

Kuosmanen $\mathrm{N}=313$

et al. discharged

education.

Highest satisfaction was with staff kindness (61.6\% satisfied) and availability (59.9\%), helpfulness of

$50 \%$

hospitalistion (58. the treatment programme $(25.6 \%)$
Older patients more satisfied with some aspects of the ward (shared bedrooms, usefulness of treatment), but less informed about ability to access own records. Men were more satisfied with staff. Patients who thought they had been prematurely discharged $(n=31)$ were less satisfied with the service. No effect of type of admission (i.e. voluntary or compulsory)

Overall mean CSQ score (22.5) showed moderate satisfaction. 73.4\% rated themselves as fairly or very satisfied. 15.5\% expressed dissatisfaction. Females $\left(\chi^{2}=7.69, \mathrm{p}=0.006\right)$, younger service users $(\mathrm{t}=2.15$, $\mathrm{p}=0.033)$, and those detained under the Mental Health Act $\left(\chi^{2}=7.56, p=0.006\right)$ were more likely to be dissatisfied. No effect of ethnicity. $66 \%$ of participants reported at least one adverse experience. Those who reported an adverse experience were more dissatisfied $(p<0.001)$ and were more likely to be detained $(\mathrm{p}<0.001)$

$>70 \%$ indicated satisfaction with services received. No effect of age, gender, education, Axis 1 diagnosis and length of stay. Number of previous admissions was negatively correlated with satisfaction (CSQ-8, $r=-0.15$

$\mathrm{p}=0.04)$. Higher satisfaction among people who were admitted voluntarily $(t=2.9, p=0.04)$.

No significant effect of age, gender or occupation. Significantly higher satisfaction was reported in patients with a diagnosis of affective illness and reactive psychosis compared to other diagnostic groups $\left(\chi^{2}=13.8\right.$, $\mathrm{p}<0.002)$

sa

Mean satisfaction score $=26.8$ (ZUF-8). No difference between the two diagnostic groups in terms of overall satisfaction. Service users with depression, and a comorbid personality disorder reported lower satisfaction than those without a personality disorder $(t=2.31, p=0.03)$. In depressed patients, severity of symptoms negatively correlated with satisfaction. No correlation between satisfaction and psychotherapy. No effect of age, gender, marital status, educational status, professional training or current occupation. No relationship with length of treatment. The number of medications correlated negatively with satisfaction (Depression: $r=-0.28, p=0.02$, schizophrenia: $r=-0.24, p=0.03$ ).

Highest satisfaction was with staff-patient relationships. Good satisfaction with help from staff and the caring nature of staff. Low satisfaction with restrictions on movement, information regarding the right to appeal, opportunities for meaningful activities and shared rooms. With regard to staff-patient relationships, service users age $45-65$ were more satisfied than those age $18-24$ (means 4.22 vs $3.8, p<0.001$ ), men were more

Finland


satisfied than women (mean 4.08 vs $3.91, \mathrm{p}=0.033$ ), and those whose current hospital stay was under two satisfied than women (mean 4.08 vs $3.91, \mathrm{p}=0.033$ ), and those whose current hospital stay was under two
weeks were more satisfied than those whose hospital stay was $1-3$ months (means 4.10 vs $3.76, \mathrm{p}=0.012$ ).

on previous $\quad$ satisfaction, but dissatisfaction expressed with some specific aspects (e.g. information regarding treatment).

research. Semi- $\quad$ Ward environment - general satisfaction was good. Safety - lowest levels of satisfaction overall. Approximately

$\mathrm{N}=6$ discharged

in qualitative

\begin{tabular}{|c|c|c|c|c|c|c|}
\hline $\begin{array}{l}\text { Lovell } \\
(1995)\end{array}$ & $\begin{array}{l}\mathrm{N}=25 \text { current } \\
\text { inpatients } \\
\text { completed } \\
\text { questionnaires. } \\
\mathrm{N}=6 \text { discharged } \\
\text { inpatients took part } \\
\text { in qualitative } \\
\text { interviews }\end{array}$ & $\begin{array}{l}\text { Acute admission } \\
\text { wards } \\
\text { England }\end{array}$ & $\begin{array}{l}\text { Mixed } \\
\text { Methods }\end{array}$ & $\begin{array}{l}\text { Developed a } \\
\text { questionnaire based } \\
\text { on previous } \\
\text { research. Semi- } \\
\text { structured interview }\end{array}$ & $\begin{array}{l}\text { Admission - general satisfaction was high, but some dissatisfaction expressed with aspects (e.g. the welcome } \\
\text { given). Qualitative interviews suggest a higher level of dissatisfaction. Treatment - Generally high } \\
\text { satisfaction, but dissatisfaction expressed with some specific aspects (e.g. information regarding treatment). } \\
\text { Ward environment - general satisfaction was good. Safety - lowest levels of satisfaction overall. Approximately } \\
72 \% \text { reported being frightened by other users }\end{array}$ & 0 \\
\hline $\begin{array}{l}\text { Middelboe } \\
\text { et al. } \\
\text { (2001) }\end{array}$ & $\begin{array}{l}\mathrm{N}=101 \text { current } \\
\text { inpatients }\end{array}$ & $\begin{array}{l}\text { Acute admission } \\
\text { ward } \\
\text { Denmark }\end{array}$ & $\begin{array}{l}\text { Descriptive } \\
\text { cross- } \\
\text { sectional }\end{array}$ & $\begin{array}{l}\text { Ward Atmosphere } \\
\text { Scale. (Moos and } \\
\text { Houts, 1968) } \\
\text { Satisfaction Scale } \\
\text { adapted from Good } \\
\text { Milieu Index }\end{array}$ & $\begin{array}{l}\text { Patient satisfaction was moderate to high and did not differ by type of unit. Aspects of ward atmosphere } \\
\text { (order and support) predicted satisfaction. Coercion was associated with significantly lower total scores on } \\
\text { the satisfaction scale }(\mathrm{t}=2.1, \mathrm{p}<0.01) \text {. }\end{array}$ & $25 \%$ \\
\hline $\begin{array}{l}\text { Muller et } \\
\text { al. (2002) }\end{array}$ & $\begin{array}{l}\mathrm{N}=135 \text { inpatients } \\
\text { at either point of } \\
\text { admission }(\mathrm{N}=60) \\
\text { or discharge } \\
(\mathrm{n}=75) \text { on either } \\
\text { open }(\mathrm{n}=153) \text { or } \\
\text { closed }(\mathrm{n}=125) \\
\text { wards }\end{array}$ & $\begin{array}{l}\text { Open/closed } \\
\text { psychiatric } \\
\text { wards } \\
\text { Germany }\end{array}$ & $\begin{array}{l}\text { Descriptive } \\
\text { cross- } \\
\text { sectional }\end{array}$ & SATQ-98 & $\begin{array}{l}\text { Average level of satisfaction was high to intermediate. } 15.3 \% \text { of patients were dissatisfied or very dissatisfied } \\
\text { with medication. General satisfaction was higher at discharge, and on the open ward }(p<0.05) \text {. Satisfaction } \\
\text { was significantly different in those that had and hadn't experienced coercion }(t=2.1, p=0.01)\end{array}$ & $75 \%$ \\
\hline $\begin{array}{l}\text { Olusina et } \\
\text { al. (2002) }\end{array}$ & $\begin{array}{l}\mathrm{N}=118 \text { inpatients } \\
\text { on day of discharge }\end{array}$ & $\begin{array}{l}\text { Two psychiatric } \\
\text { wards, general } \\
\text { hospital. } \\
\text { Nigeria }\end{array}$ & $\begin{array}{l}\text { Descriptive } \\
\text { cross- } \\
\text { sectional }\end{array}$ & $\begin{array}{l}\text { Patient Care } \\
\text { Assessment } \\
\text { Questionnaire - } \\
\text { developed based on } \\
\text { previous research. }\end{array}$ & $\begin{array}{l}\text { Dissatisfaction was related to curtailment of freedom. Highest satisfaction ratings were related to the staff- } \\
\text { patient relationship. Age was associated with the perception of access to staff }(\mathrm{f}=3.3, \mathrm{p}<0.01) \text {, with service } \\
\text { users aged above } 25 \text { reporting higher satisfaction. Females were more likely to be satisfied with ward } \\
\text { environment than men }(\mathrm{t}=2.97, \mathrm{p}<0.005) \text {. }\end{array}$ & $75 \%$ \\
\hline $\begin{array}{l}\text { Prince } \\
(2006)\end{array}$ & $\begin{array}{l}\mathrm{N}=315 \text { former } \\
\text { inpatients with a } \\
\text { diagnosis of } \\
\text { Schizophrenia } \\
\text { within } 72 \mathrm{~h} \text { of } \\
\text { discharge }\end{array}$ & $\begin{array}{l}\text { Inpatient } \\
\text { psychiatric } \\
\text { wards, four } \\
\text { general } \\
\text { hospitals. } \\
\text { USA }\end{array}$ & $\begin{array}{l}\text { Descriptive } \\
\text { cross- } \\
\text { sectional }\end{array}$ & $\begin{array}{l}\text { Structured } \\
\text { assessment, ratings } \\
\text { of satisfaction made } \\
\text { on a 5-point scale }\end{array}$ & $\begin{array}{l}\text { Black people were more likely to report higher satisfaction }(\mathrm{OR}=0.56, \mathrm{p}<0.02) \text {. Highest satisfaction was } \\
\text { associated with staff effort. No effect of level of functioning }\end{array}$ & $25 \%$ \\
\hline $\begin{array}{l}\text { Ricketts } \\
(1996)\end{array}$ & $\begin{array}{l}\mathrm{N}=55 \text { former } \\
\text { inpatients within } 2 \\
\text { days of discharge }\end{array}$ & $\begin{array}{l}\text { Psychiatric } \\
\text { admission ward. } \\
\text { England }\end{array}$ & $\begin{array}{l}\text { Mixed } \\
\text { methods }\end{array}$ & $\begin{array}{l}\text { Client Satisfaction } \\
\text { Questionnaire (CSQ) }\end{array}$ & $\begin{array}{l}\text { Mean CSQ score }=22.8 \text {. Significant correlation between general satisfaction and satisfaction with nursing } \\
\text { communication. Lowest satisfaction was with the services ability to meet needs }\end{array}$ & $25 \%$ \\
\hline $\begin{array}{l}\text { Rose et al. } \\
(2015)\end{array}$ & $\begin{array}{l}\mathrm{N}=37 \text { service } \\
\text { users discharged } \\
\text { from inpatient } \\
\text { services within the } \\
\text { last } 2 \text { years }\end{array}$ & $\begin{array}{l}\text { Psychiatric } \\
\text { wards. } \\
\text { England }\end{array}$ & $\begin{array}{l}\text { Qualitative } \\
\text { - focus } \\
\text { groups }\end{array}$ & $\begin{array}{l}\text { Topic guide, } \\
\text { thematic analysis }\end{array}$ & $\begin{array}{l}\text { The ward was felt to be untherapeutic and the staff unavailable. Lack of freedom to leave the ward led to } \\
\text { frustration, and coercion was experienced as a result. }\end{array}$ & $50 \%$ \\
\hline $\begin{array}{l}\text { Ruggeri et } \\
\text { al. (2003) }\end{array}$ & $\begin{array}{l}\mathrm{N}=404 \text { current } \\
\text { inpatients with a } \\
\text { diagnosis of } \\
\text { schizophrenia }\end{array}$ & $\begin{array}{l}\text { Psychiatric } \\
\text { wards. } \\
\text { The } \\
\text { Netherlands, } \\
\text { Denmark, UK, } \\
\text { Spain, Italy }\end{array}$ & $\begin{array}{l}\text { Descriptive } \\
\text { cross- } \\
\text { sectional }\end{array}$ & $\begin{array}{l}\text { Verona Service } \\
\text { Satisfaction Scale } \\
\text { (VSS-EU; Knudsen } \\
\text { et al., 2000) }\end{array}$ & $\begin{array}{l}\text { Satisfaction differed across the research sites. Living in London/Santander, being retired/unemployed, having } \\
\text { a high number of hospital admissions, high levels of psychopathology, unmet needs and poor quality of life } \\
\text { were all associated with low total service satisfaction. }\end{array}$ & $25 \%$ \\
\hline
\end{tabular}

et al.

$\mathrm{N}=101$ current

Acute admission Descriptive

ward

cross-

Satisfaction Scale

dapted from Good

ilieu Index

Average level of satisfaction was high to intermediate. 15.3\% of patients were dissatisfied or very dissatisfied with medication. General satisfaction was higher at discharge, and on the open ward $(p<0.05)$. Satisfaction was significantly different in those that had and hadn't experienced coercion $(t=2.1, p=0.01)$ previous research.

Structure assessment, ratings of satisfaction made Schizophrenia discharge inpatients within 2 users discharge from inpatien ervices within the $\mathrm{N}=404$ curren inpatients with diagnosis of

Netherlands, Spain, Italy

Patient satisfaction was moderate to high and did not differ by type of unit. Aspects of ward atmosphere (order and support) predicted satisfaction. Coercion was associated with significantly lower total scores on

Black people were more likely to report higher satisfaction $(\mathrm{OR}=0.56, \mathrm{p}<0.02)$. Highest satisfaction was crosson a 5-point scale

Client Satisfaction

The ward was felt to be untherapeutic and the staff unav 


\begin{tabular}{|c|c|c|c|c|c|c|}
\hline $\begin{array}{l}\text { Shiva et al. } \\
\text { (2009) }\end{array}$ & $\begin{array}{l}\mathrm{N}=188 \text { current } \\
\text { inpatients }\end{array}$ & $\begin{array}{l}\text { Psychiatric } \\
\text { hospital. } \\
\text { USA }\end{array}$ & $\begin{array}{l}\text { Descriptive } \\
\text { cross- } \\
\text { sectional }\end{array}$ & $\begin{array}{l}\text { Inpatient } \\
\text { Satisfaction } \\
\text { Questionnaire } \\
\text { (Shiva et al., 2009) }\end{array}$ & Patients with a diagnosis of a psychotic disorder were more satisfied than those without & $75 \%$ \\
\hline $\begin{array}{l}\text { Smith et al. } \\
(2014)\end{array}$ & $\begin{array}{l}\mathrm{N}=129 \text { inpatients } \\
\text { in the week prior to } \\
\text { discharge }\end{array}$ & $\begin{array}{l}\text { Three mental } \\
\text { health wards, } \\
\text { and one private } \\
\text { hospital ward } \\
\text { Ireland }\end{array}$ & $\begin{array}{l}\text { Descriptive } \\
\text { cross- } \\
\text { sectional }\end{array}$ & $\begin{array}{l}\text { CSQ-8. } \\
\text { McArthur Admission } \\
\text { Experience } \\
\text { Interview (Monahan } \\
\text { et al., 1995) }\end{array}$ & $\begin{array}{l}\text { Mean CSQ-8 score }=24.5 \text { ('good'). No effect of age, gender or number or length of admissions on the level of } \\
\text { reported satisfaction. Satisfaction significantly higher in those admitted voluntarily, than those involuntarily } \\
\text { admitted }(\mathrm{t}=-3.9, \mathrm{p}<0.001) \text {. Insight positively correlated with satisfaction }(\mathrm{re}=0.24, \mathrm{p}=0.01) \text {, as did } \\
\text { functioning }(\mathrm{rs}=0.25, \mathrm{p}=0.01 \text { ). No effect of type of disorder, or severity of psychotic symptoms. Patients } \\
\text { with comorbid drug misuse reported lower satisfaction than those without }(\mathrm{t}=-2.86, \mathrm{p}=0.01) \text {. Strength of } \\
\text { the therapeutic relationship (psychiatrist) was positively correlated with satisfaction }(\mathrm{rs}=0.63, \mathrm{p}<0.001 \text { ). } \\
16 \% \text { of service users reported some form of physical coercion, and reported significantly lower satisfaction } \\
\text { than those who did not }(\mathrm{t}=-2.94, \mathrm{p}=0.01 \text { ) }\end{array}$ & $50 \%$ \\
\hline $\begin{array}{l}\text { Sorgaard } \\
(2007)\end{array}$ & $\begin{array}{l}\mathrm{N}=189 \text { current } \\
\text { inpatients }\end{array}$ & $\begin{array}{l}\text { Three closed } \\
\text { acute wards } \\
\text { Norway }\end{array}$ & $\begin{array}{l}\text { Descriptive } \\
\text { cross- } \\
\text { sectional }\end{array}$ & SPRI & Committed patients were more dissatisfied with treatment $\left(\chi^{2}=7.62, p=0.022\right)$ & $75 \%$ \\
\hline $\begin{array}{l}\text { Soergaard } \\
\text { et al. } \\
\text { (2008) }\end{array}$ & $\begin{array}{l}\mathrm{N}=117 \text { first-time } \\
\text { inpatients at } \\
\text { discharge }\end{array}$ & $\begin{array}{l}\text { Psychiatric } \\
\text { ward } \\
\text { Norway }\end{array}$ & $\begin{array}{l}\text { Prospective } \\
\text { cross- } \\
\text { sectional }\end{array}$ & SPRI & $\begin{array}{l}\text { Female patients tended to report more neutral aspects of satisfaction then men }(p=0.047) \text {. Age was also } \\
\text { associated with positive }(p<0.01) \text { satisfaction scores. }\end{array}$ & $25 \%$ \\
\hline $\begin{array}{l}\text { Stewart et } \\
\text { al. (2015) }\end{array}$ & $\begin{array}{l}\mathrm{n}=119 \text { current } \\
\text { inpatients }\end{array}$ & $\begin{array}{l}13 \text { acute and } 3 \\
\text { PICU wards } \\
\text { across } 3 \\
\text { hospital sites } \\
\text { England }\end{array}$ & $\begin{array}{l}\text { Qualitative } \\
\text { - semi } \\
\text { structured } \\
\text { interviews }\end{array}$ & Thematic analysis & $\begin{array}{l}\text { Staff duties - appreciation of staff roles and duties. Increased sense of trust and safety associated with } \\
\text { perceptions of nurses as capable. Staff disposition - quality of care related to nurses' attitudes towards } \\
\text { patients and their work. Value of being treated as an individual. Control - necessary to maintain order, could } \\
\text { be overwhelming. Therapeutic ward environment - boredom, too much paperwork for staff. Changes to staff } \\
\text { could be disruptive. Communication and engagement - felt one way, could not initiate. Consistency - rule } \\
\text { keeping inconsistently applied }\end{array}$ & $50 \%$ \\
\hline $\begin{array}{l}\text { Sweeney et } \\
\text { al. (2014) }\end{array}$ & $\begin{array}{l}\mathrm{N}=247 \text { current } \\
\text { inpatients - } \\
\text { quantitative arm, } \\
\mathrm{N}=14 \text { current } \\
\text { inpatients - } \\
\text { qualitative arm. }\end{array}$ & $\begin{array}{l}16 \text { psychiatric } \\
\text { inpatient wards } \\
\text { England }\end{array}$ & $\begin{array}{l}\text { Mixed } \\
\text { methods }\end{array}$ & $\begin{array}{l}\text { Client Satisfaction } \\
\text { Questionnaire } \\
\text { (CSQ). Thematic } \\
\text { analysis }\end{array}$ & $\begin{array}{l}\text { Mean CSQ-8 score }=21.0 \text {. Deprivation of freedom and lack of autonomy resulted in negative dynamics } \\
\text { between patients and staff. Lack of staff visibility appeared to have an effect on SUs, ward atmosphere and } \\
\text { therapeutic alliance. No effect of recovery. Therapeutic alliance and peer support were related to satisfaction } \\
\text { levels }\end{array}$ & $75 \%$ \\
\hline $\begin{array}{l}\text { Wallace et } \\
\text { al. (1999) }\end{array}$ & $\begin{array}{l}\mathrm{N}=23 \text { former } \\
\text { inpatients, } \\
\text { discharged in the } \\
\text { year before the } \\
\text { study }\end{array}$ & $\begin{array}{l}\text { Inpatient unit, } \\
\text { Department of } \\
\text { Psychiatry } \\
\text { Canada }\end{array}$ & $\begin{array}{l}\text { Qualitative } \\
\text { - focus } \\
\text { groups }\end{array}$ & Thematic analysis & $\begin{array}{l}\text { Continuity was seen as important. There was uncertainty regarding the need for medications. Inactivity and a } \\
\text { negative environment affected the therapeutic effect of services. Need for individualised care. Need for staff } \\
\text { to be accessible and give information. }\end{array}$ & $50 \%$ \\
\hline $\begin{array}{l}\text { Zahid et al. } \\
(2010)\end{array}$ & $\begin{array}{l}\mathrm{N}=130 \text { recently } \\
\text { discharged former } \\
\text { inpatients with a } \\
\text { diagnosis of } \\
\text { schizophrenia }\end{array}$ & $\begin{array}{l}\text { One unit of the } \\
\text { Psychological } \\
\text { Medicine } \\
\text { Hospital } \\
\text { Kuwait }\end{array}$ & $\begin{array}{l}\text { Descriptive } \\
\text { cross- } \\
\text { sectional }\end{array}$ & VSS-EU & $\begin{array}{l}\text { General satisfaction was good. At least } 2 / 3 \text { expressed overall satisfaction. Lowest endorsements of } \\
\text { satisfaction were for information and types of intervention. Women reported higher satisfaction than men } \\
(\mathrm{t}=2.9, \mathrm{p}<0.02) \text {. Divorced rated satisfaction higher than married }(\mathrm{F}=5.2, \mathrm{p}<0.007) \text {. Age correlated with } \\
\text { satisfaction with 'types of intervention' }(\mathrm{r}=0.22, \mathrm{p}<0.01) \text {. No effect of years of education. Those who rated } \\
\text { themselves as having enough money to enjoy themselves scored significantly higher on satisfaction scale } \\
(\mathrm{p}<0.03)\end{array}$ & $75 \%$ \\
\hline $\begin{array}{l}\text { Zendjidjian } \\
\text { et al. } \\
\text { (2014) }\end{array}$ & $\begin{array}{l}\mathrm{N}=270 \text { inpatients } \\
\text { on day of discharge }\end{array}$ & $\begin{array}{l}\text { Psychiatric } \\
\text { departments of } \\
\text { two University } \\
\text { teaching } \\
\text { hospitals } \\
\text { France }\end{array}$ & $\begin{array}{l}\text { Descriptive } \\
\text { cross- } \\
\text { sectional }\end{array}$ & $\begin{array}{l}\text { Ssatispsy-22 } \\
\text { (Zendjidjian et al., } \\
\text { 2014) }\end{array}$ & $\begin{array}{l}\text { Satisfaction was positively associated with marital status (satisfaction was higher in people who were in a } \\
\text { couple, } p<0.001) \text {, voluntary admission }(p=0.022) \text {, absence of seclusion }(p=0.001) \text {, more previous } \\
\text { admissions }(p=0.05) \text {, better therapeutic alliance }(p=0.001) \text { and better functioning }(p=0.03) \text {. }\end{array}$ & $50 \%$ \\
\hline
\end{tabular}




\subsection{Quality assessment}

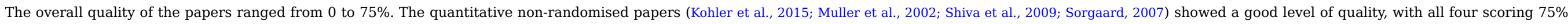

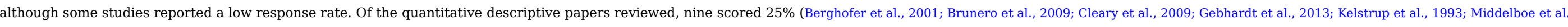

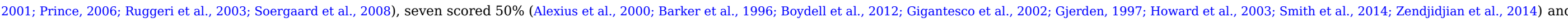

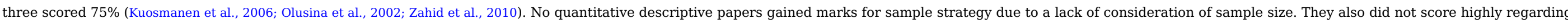

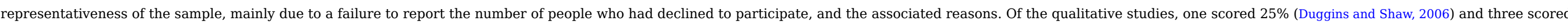

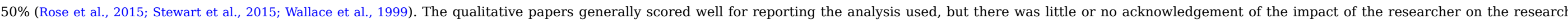
process. The four mixed methods papers scored 0\% (Lovell, 1995), 25\% (Sweeney et al., 2014), 50\% (Greenwood et al., 1999) and 75\% (Ricketts, 1996) respectively.

\subsection{General satisfaction}

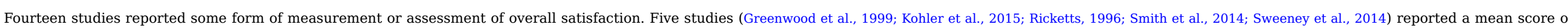

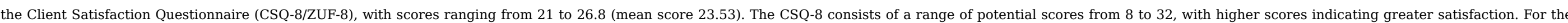

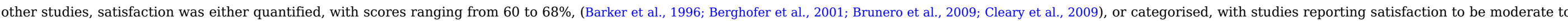
high (Middelboe et al., 2001; Muller et al., 2002) or simply 'good' (Zahid et al., 2010).

\subsection{Definition and measurement of satisfaction}

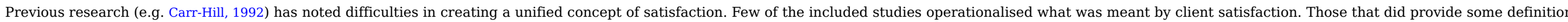

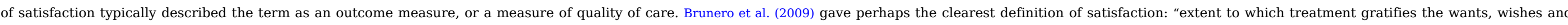

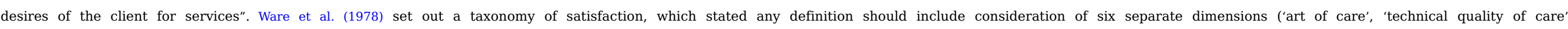

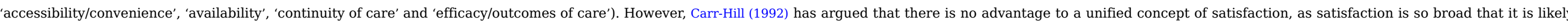
to have a different meaning to each service user.

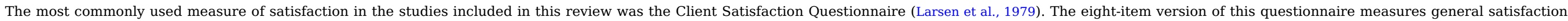

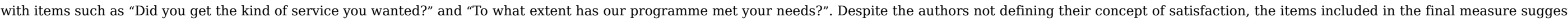

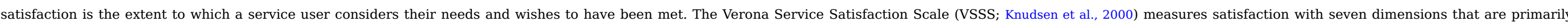

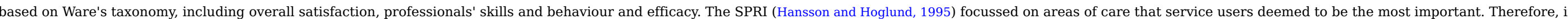
seems that the most commonly used questionnaires are based on a range of factors, but have in common a focus on the quality of care on offer, and a rating of overall satisfaction.

\subsection{Factors relating to satisfaction}

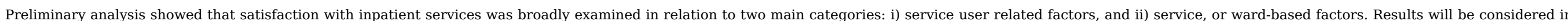
relation to these categories.

\subsection{Service user related factors}

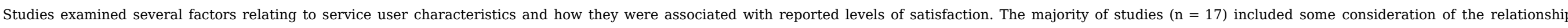

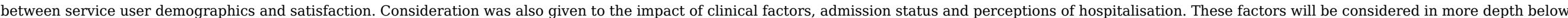

\subsubsection{Service user demographics}

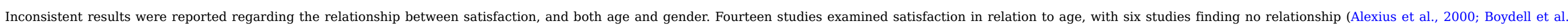

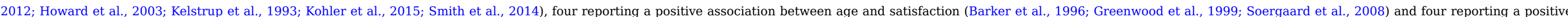




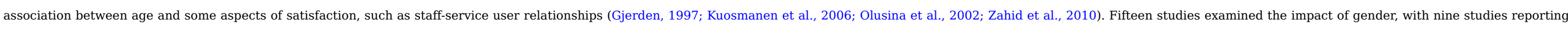

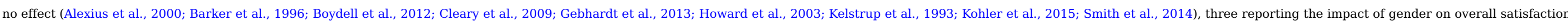

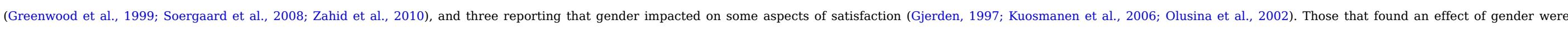

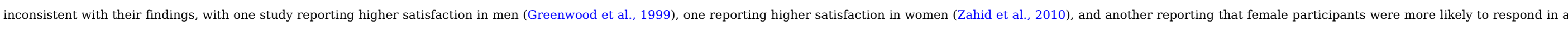

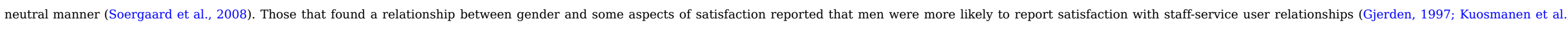

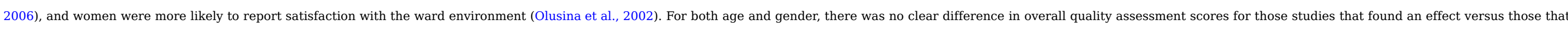

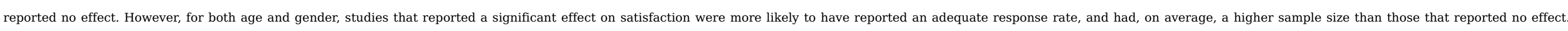
Studies that reported an association between age and satisfaction were more likely to be based in the UK, with those that reported no effect largely based in continental Europe.

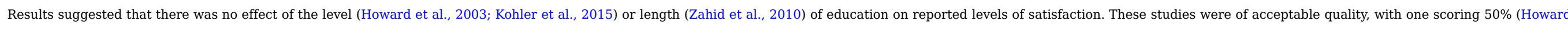

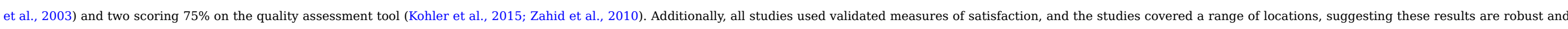
valid.

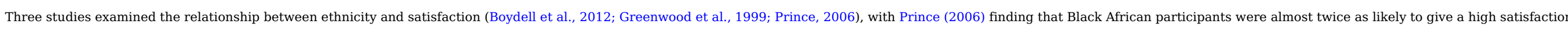

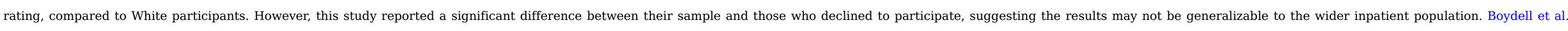

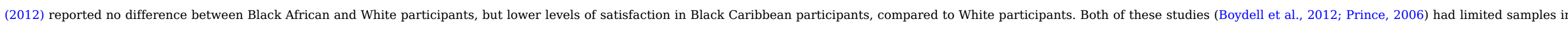

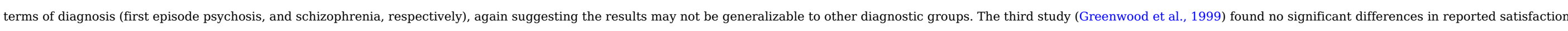

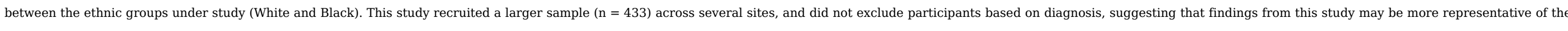

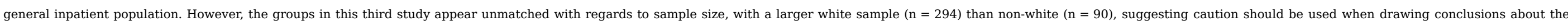
impact of ethnicity from this study.

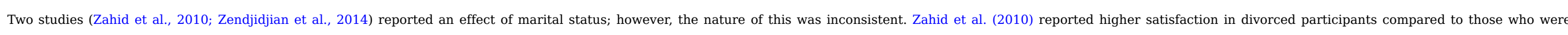

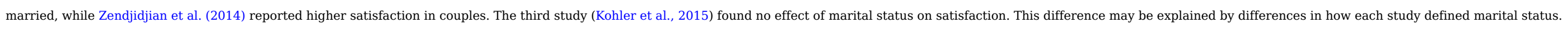

\subsubsection{Admission status}

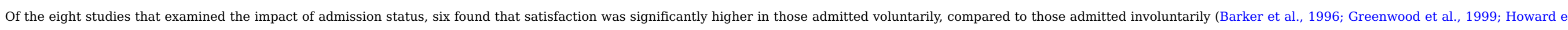

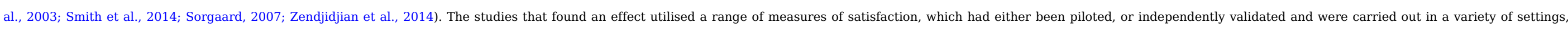

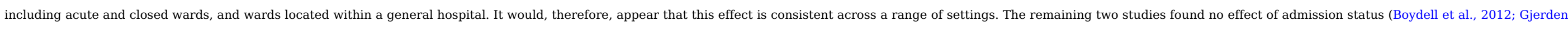

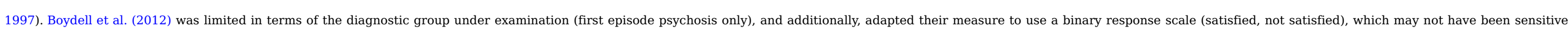
enough to detect differences between the groups. Gjerden (1997) recruited only from an open ward, and therefore results may not be generalizable to the wider inpatient population.

\subsubsection{Perceptions of need for hospitalisation and illness}

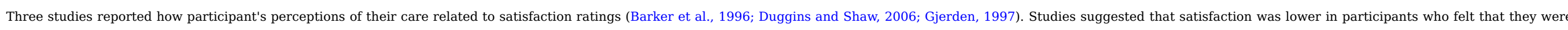

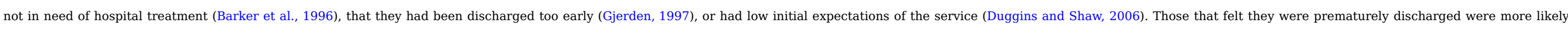

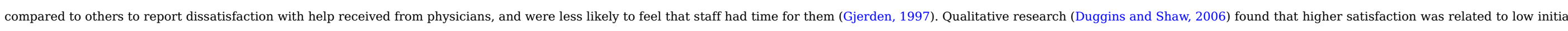

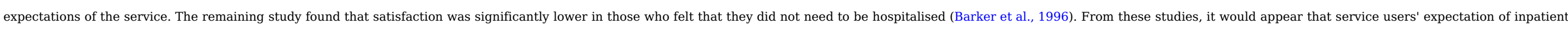

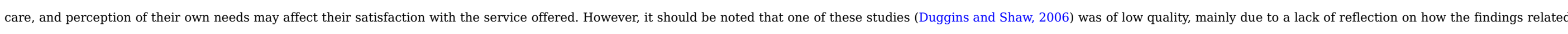
to the researchers' own views and experiences, and the research context. The remaining two studies had higher overall scores for quality, suggesting the results may be more acceptable.

\subsubsection{Clinical factors}

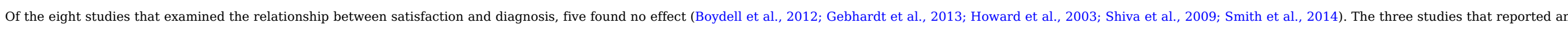




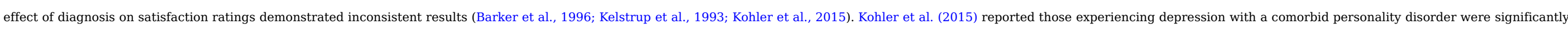

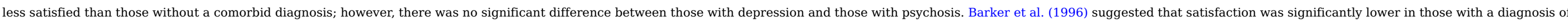

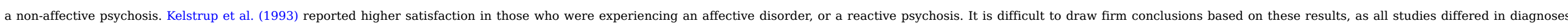

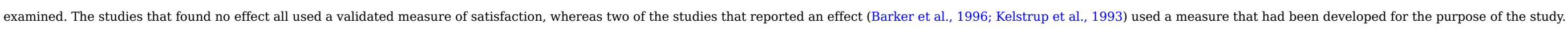

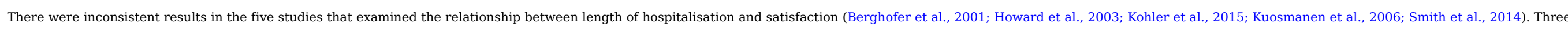

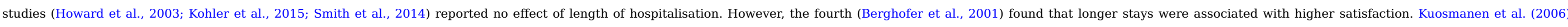

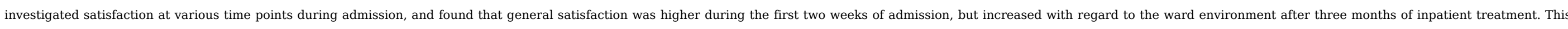

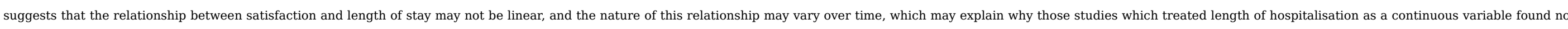
effect.

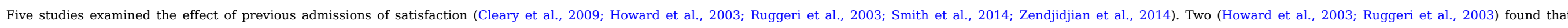

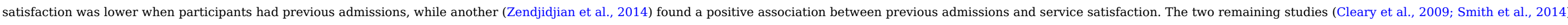

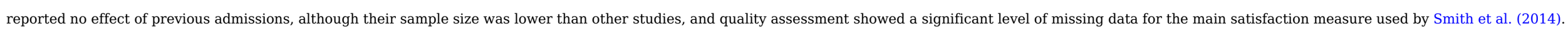

\subsection{Service related factors}

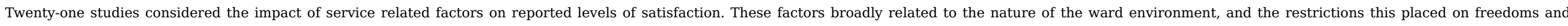
activities, the opportunity to seek understanding and support from staff and other service users. Full analysis of these factors and their relationship to satisfaction is provided below.

\subsubsection{Open/closed ward}

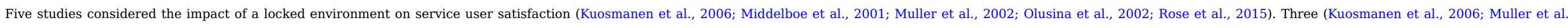

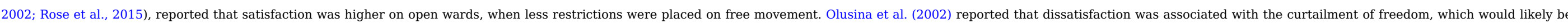

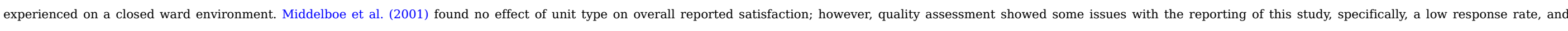
significant differences between those who participated and those who did not. The other studies reported here were of higher quality, suggesting their results may be of higher validity.

\subsubsection{Ward environment}

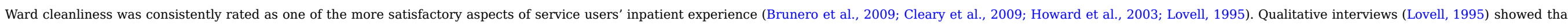

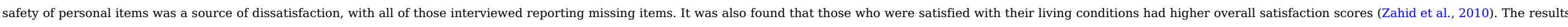
relating to ward environment did show consistency across a range of settings, suggesting they may be generalizable to the general inpatient population.

\subsubsection{Information giving}

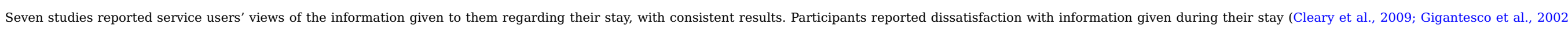

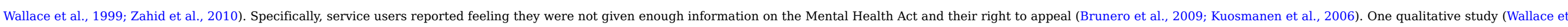

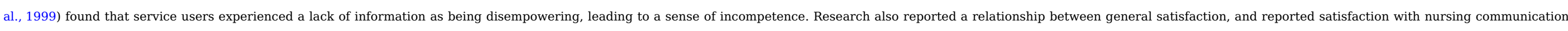

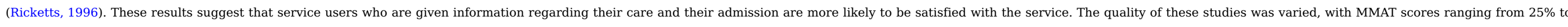
$75 \%$. However, the consistency of these results suggests they may be generalizable to the wider inpatient population.

\subsubsection{Coercion and safety}

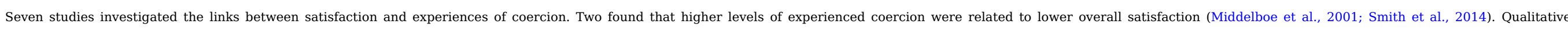

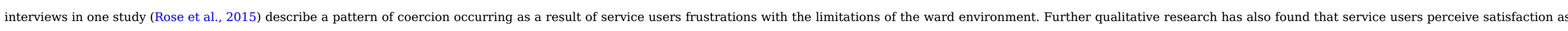

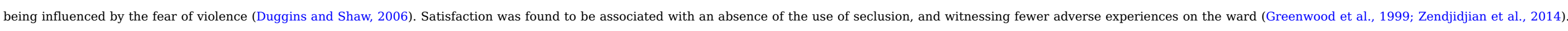




\subsubsection{Staff}

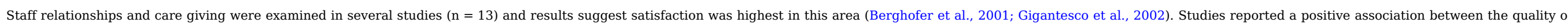

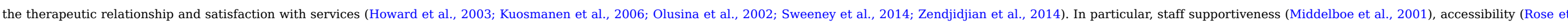

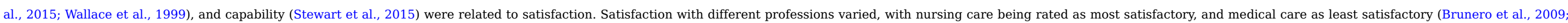

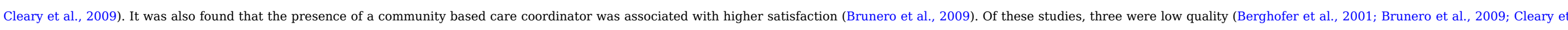

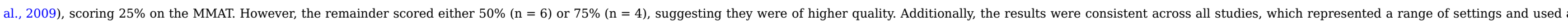
variety of measures of satisfaction, suggesting these results are valid and reliable.

\section{Discussion}

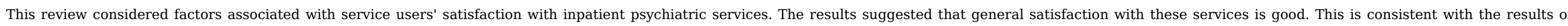

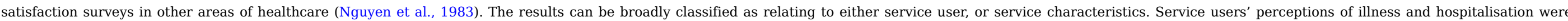

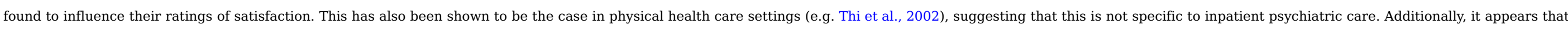

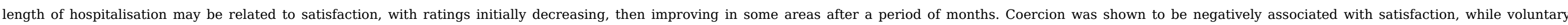
admission was positively associated with satisfaction. Satisfaction was reported to be higher on open than closed wards, possibly due to the restrictions placed on freedom on closed wards.

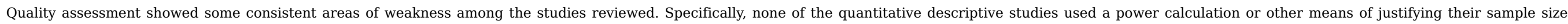

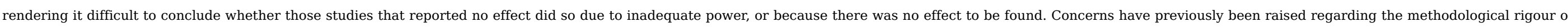

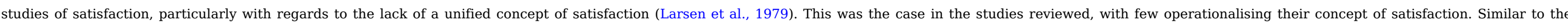

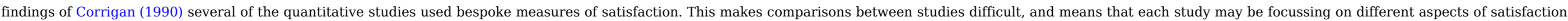

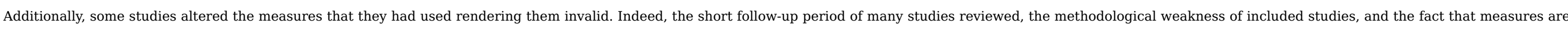
inconsistently used suggest that little has been done to address methodological issues in the period since Corrigan's original review.

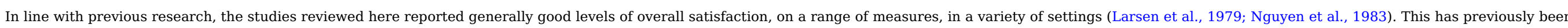

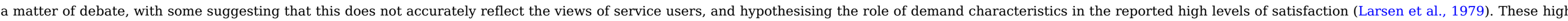

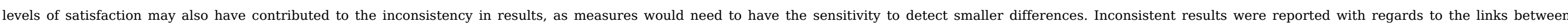

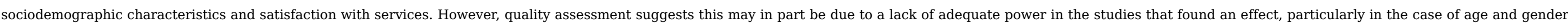

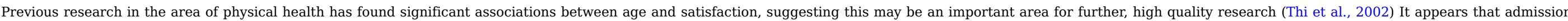

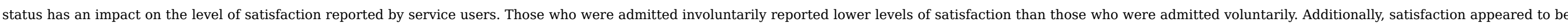

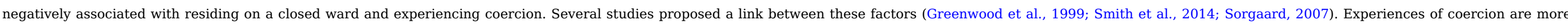

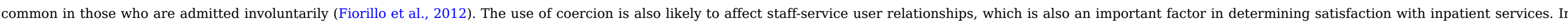

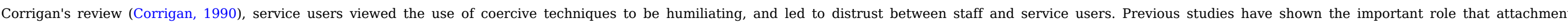

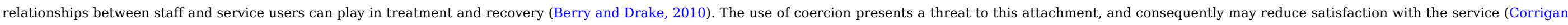

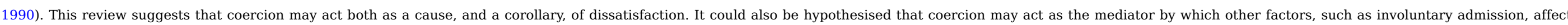

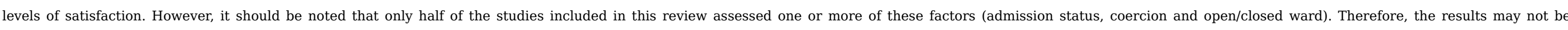
generalizable to wider populations. However, the majority of studies suggested that these factors were related to satisfaction, and those that did not appeared to be of lower quality.

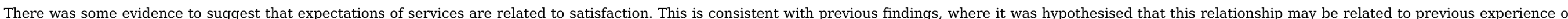




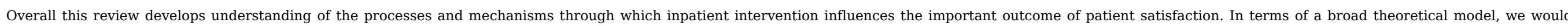

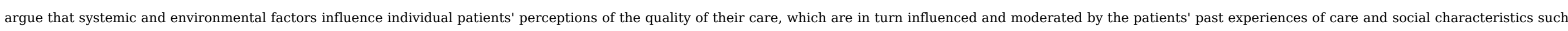

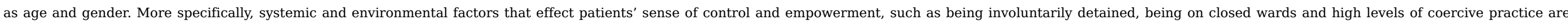

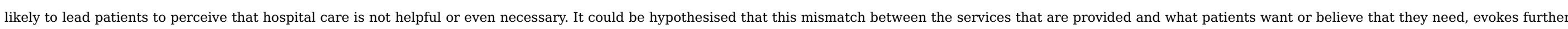

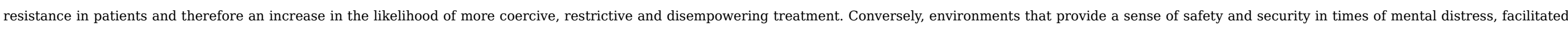

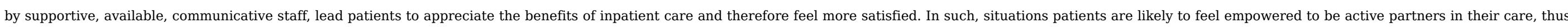

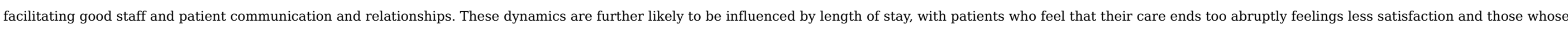
stay is extended perhaps due to reasons beyond their control, feeling that care is unnecessarily long and restrictive.

\subsection{Strengths and limitations of the review}

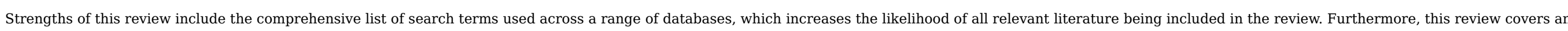

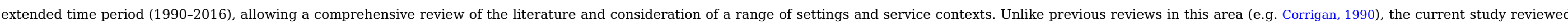

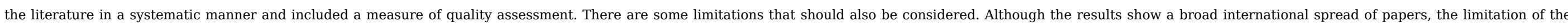

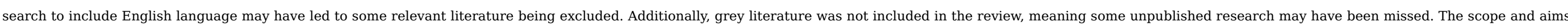

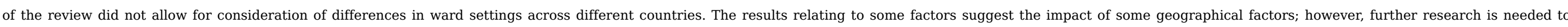
understand the mechanism by which this occurs.

\subsection{Areas for further research}

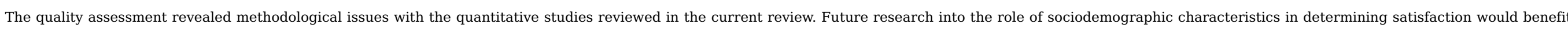

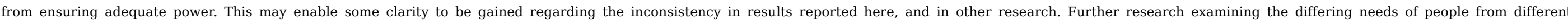

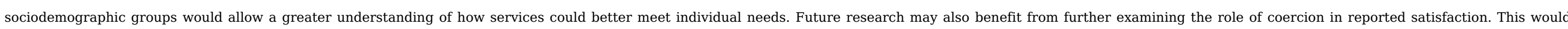

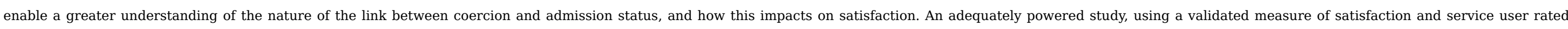

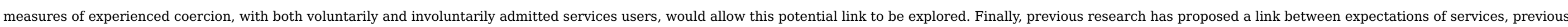

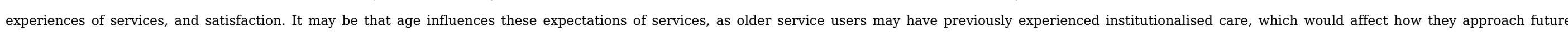

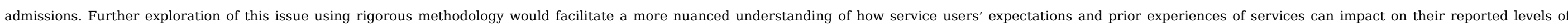
satisfaction. Further research should consider how satisfaction could be clearly operationalised. Consideration should be given to the use of standardised measures of satisfaction.

\subsection{Clinical implications}

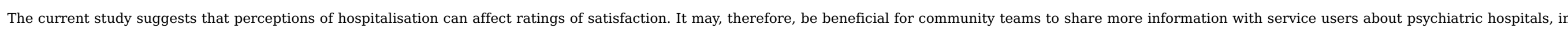

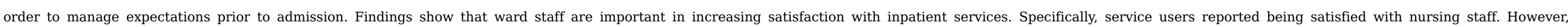

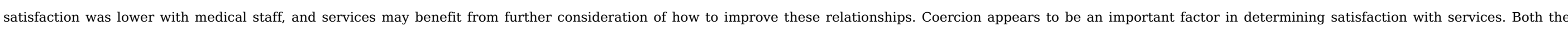

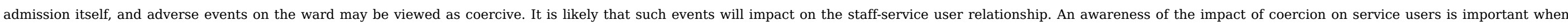
considering the need for post-incident support. By providing appropriate support to service users, staff may be able to repair ruptures in the therapeutic relationship.

\section{References}

Al-Abri R. and Al-Balushi A., Patient satisfaction survey as a tool towards quality improvement, Oman Med. J. 29 (1), 2014, 3-7, http://dx.doi.org/10.5001/omj.2014.02.

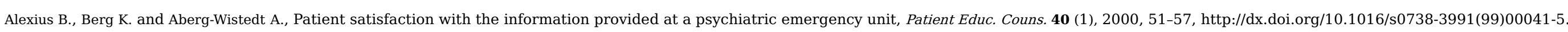




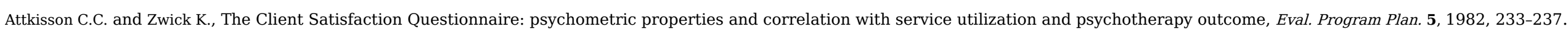

Barker D.A., Shergill S.S., Higginson I. and Orrell M.W., Patients' views towards care received from psychiatrists, Br. J. Psychiatry 168 (5), 1996, 641-646, http://dx.doi.org/10.1192/bjp.168.5.641.

Barnett-Page E. and Thomas J., Methods for the synthesis of qualitative research: a critical review, BMC Med. Res. Methodol. 9 (59), 2009.

BBC News, Mental Health Patients Forced to Travel Miles for Care, 2014, Retrieved from bbc.co.uk/news/uk-27285555.

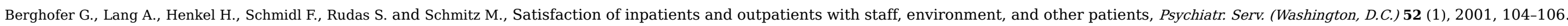

Berry K. and Drake R., Attachment theory in psychiatric rehabilitation: informing clinical practice, Adv. Psychiatric Treat. 16 (4), $2010,308-315$.

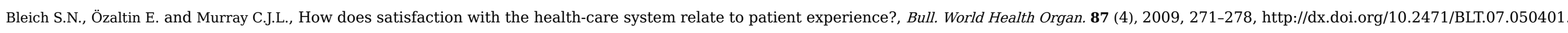

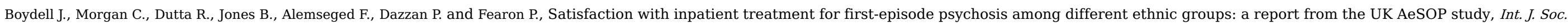
psychiatry 58 (1), 2012, 98-105.

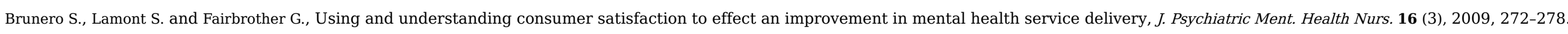

Carr-Hill R.A., The measurement of patient satisfaction, J. Public Health 14 (3), 1992, 236-249.

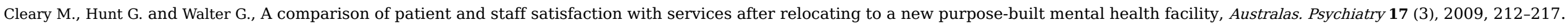

Cohen J., A coefficient of agreement for nominal scales, Educ. Psychol. Meas. 20 (1), 1960, 37-46, http://dx.doi.org/10.1177/001316446002000104.

Corrigan P.W., Consumer satisfaction with institutional and community care, Community Ment. Health J. 26 (2), 1990, 151-165, http://dx.doi.org/10.1007/BF00752392.

Druss B.G., Rosenheck R.A. and Stolar M., Patient satisfaction and administrative measures as indicators of the quality of mental health care, Psychiatr. Serv. 50 (8), 1999, 1053-1058.

Duggins R. and Shaw I., Examining the concept of patient satisfaction in patients with a diagnosis of schizophrenia: a qualitative study, Psychiatr. Bull. 30 (4), $2006,142-145$.

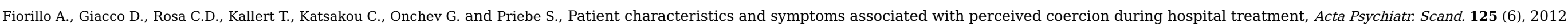
460-467, http://dx.doi.org/10.1111/j.1600-0447.2011.01809.x.

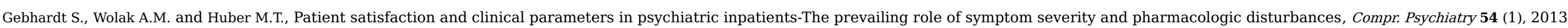
53-60.

Gigantesco A., Picardi A., Chiaia E., Balbi A. and Morosini P., Patients' and relatives' satisfaction with psychiatric services in a large catchment area in Rome, Eur. Psychiatry 17 (3), $2002,139-147$.

Gilburt H., Mental Health under Pressure, 2015.

Gjerden P., A survey of patient satisfaction as a means of evaluating quality of care in an open psychiatric ward, Nordic J. Psychiatry 51 (4), 1997, 235-242.

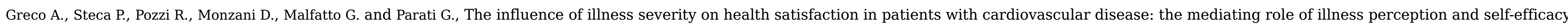
beliefs, Behav. Med. 41 (1), 2015, 9-17.

Greenwood N., Key A., Burns T., Bristow M. and Sedgwick P., Satisfaction with in-patient psychiatric services - relationship to patient and treatment factors, Br. J. Psychiatry 174, 1999, 159-163,

http://dx.doi.org/10.1192/bjp.174.2.159.

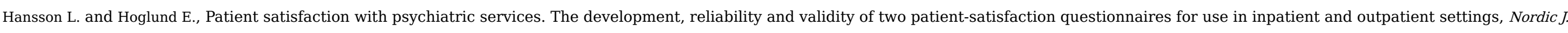
Psychiatry 49, 1995, 257-262.

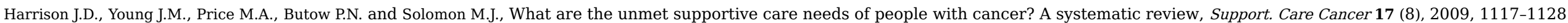




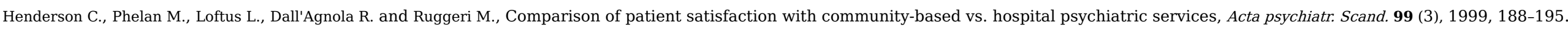

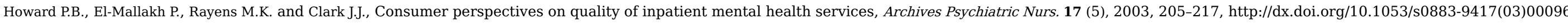
7.

Jerrell J.M., Psychometrics of the MHSIP adult consumer survey, J. Behav. Health Serv. Res. 33, 2006, 483-488.

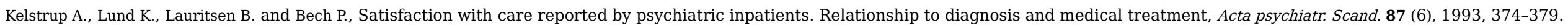

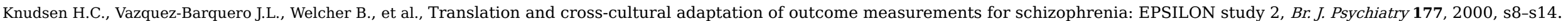

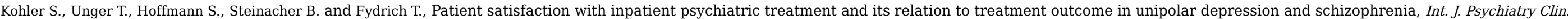
Pract. 19 (2), 2015, 119-123

Kuosmanen L., Hatonen H., Jyrkinen A.R., Katajisto J. and Valimaki M., Patient satisfaction with psychiatric inpatient care, J. Adv. Nurs. 55 (6), $2006,655-663$.

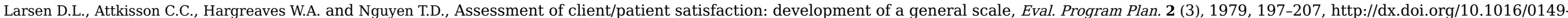
7189(79)90094-6.

Lovell K., User satisfaction with in-patient mental health services, J. psychiatric Ment. health Nurs. 2 (3), 1995, 143-150.

Medical Research Council, A Framework for the Development and Evaluation of RCTs for Complex Interventions to Improve Health, 2000, MRC; London.

Medical Research Council, Developing and Evaluating Complex Interventions: New Guidance, 2008, MRC; London.

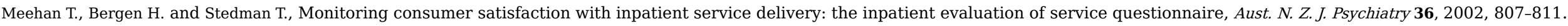

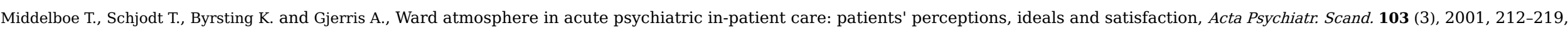

http://dx.doi.org/10.1034/j.1600-0447.2001.00102.x.

Moher D., Liberati A., Tetzlaff J., Altman D.G. and The P.G., Preferred reporting items for systematic reviews and meta-analyses: the PRISMA statement, PLoS Med. 6 (7), 2009, e1000097, http://dx.doi.org/10.1371/journal.pmed.1000097.

Monahan J., Hoge S.K., Lidz C., et al., Coercion and commitment: understanding involuntary mental hospital admissions, Int. J. Law Psychiatry. 18, 1995 , $249-263$.

Moos R.H. and Houts P.S., Assessment of the social atmosphere of psychiatric wards, J. Abnorm. Psychol. 73, 1968, 595-604.

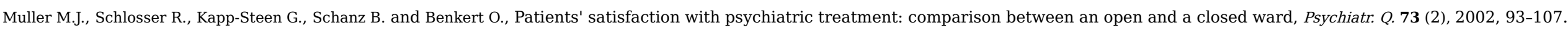

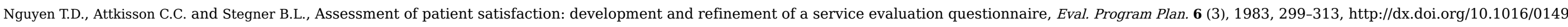
7189(83)90010-1.

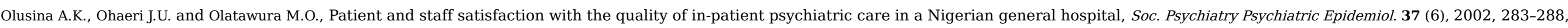
http://dx.doi.org/10.1007/s00127-002-0548-5.

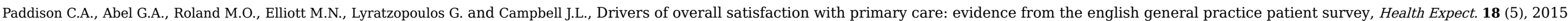
1081-1092.

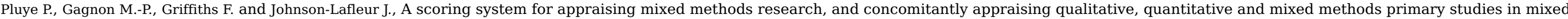
studies reviews, Int. J. Nurs. Stud. 46 (4), 2011, 529-546, http://dx.doi.org/10.1016/j.ijnurstu.2009.01.009.

Prince J.D., Determinants of care satisfaction among inpatients with schizophrenia, Community Ment. Health J. 42 (2), 2006, 189-196. 


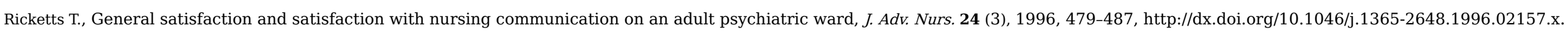

Rose D., Evans J., Laker C. and Wykes T., Life in acute mental health settings: experiences and perceptions of service users and nurses, Epidemiol. Psychiatric Sci. 24 (1), 2015, 90-96.

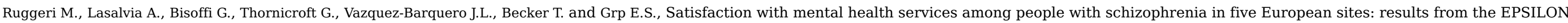
study, Schizophr. Bull. 29 (2), 2003, 229-245.

Sammut R. and Leff J., The effect of reprovision on the acute services, In: Leff J., (Ed), Care in the Community: Illusion or Reality, 1995, Wiley; Chicester.

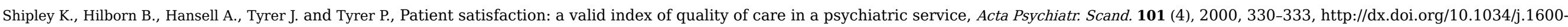
0447.2000.101004330.x.

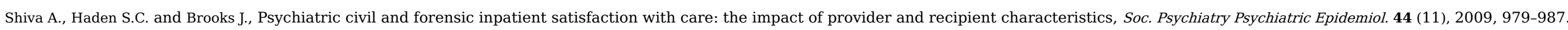

Smith D., Roche E., O'Loughlin K., Brennan D., Madigan K., Lyne J. and O'Donoghue B., Satisfaction with services following voluntary and involuntary admission, J. Ment. Health 23 (1), 2014, 38-45, http://dx.doi.org/10.3109/09638237.2013.841864.

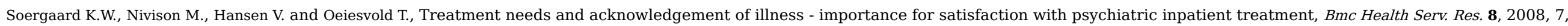
http://dx.doi.org/10.1186/1472-6963-8-103.

Sorgaard K.W., Satisfaction and coercion among voluntary, persuaded/pressured and committed patients in acute psychiatric treatment, Scand. J. Caring Sci. 21 (2), 2007 , 214-219.

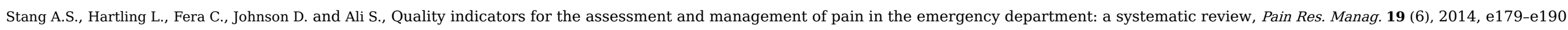

Stewart D., Burrow H., Duckworth A., Dhillon J., Fife S., Kelly S. and Bowers L., Thematic analysis of psychiatric patients' perceptions of nursing staff, Int. J. Ment. Health Nurs. 24 (1), 2015, 82-90, http://dx.doi.org/10.1111/inm.12107.

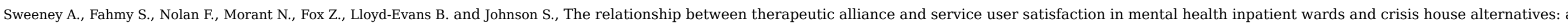
cross-sectional study, PLOS ONE $\mathbf{9}$ (7), 2014.

Thi P.L.N., Briancon S., Empereur F., Guillemin F., et al., Factors determining inpatient satisfaction with care, Soc. Sci. Med. 54 (4), 2002, 483-504.

USA Today, Cost of Not Caring: Nowhere to Go - the Financial and Human Toll for Neglecting the Mentally Ill, 2014.

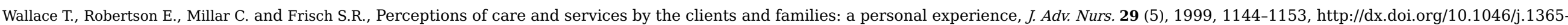
2648.1999.00996.x.

Ware J.E., Jr., Davies-Avery A. and Stewart A.L., The measurement and meaning of patient satisfaction: a review of the literature, Health Med. Serv. 1., $1978,1-15$.

Williams B. and Wilkinson G., Patient satisfaction in mental health care. Evaluating an evaluative method, Br. J. Psychiatry 166 (5), 1995, 559-562.

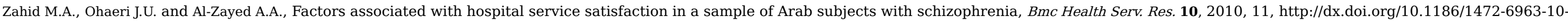
294.

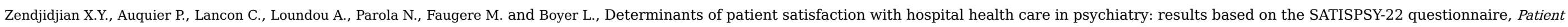
Prefer. Adherence 8, 2014, 1457-1464.

\section{Queries and Answers}

Query: The country name has been inserted for the affiliation and corresponding author field. Please check, and correct if necessary.

Answer: United Kingdom 


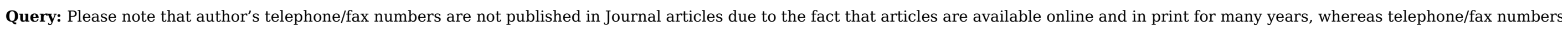
are changeable and therefore not reliable in the long term.

Answer: OK

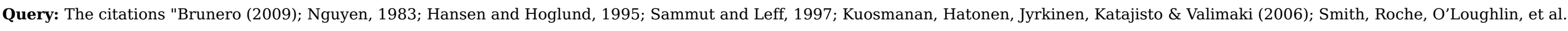
(2014); Sweeney, Fahmy, Nolan, et al. (2015)" have been changed to match the author name/date in the reference list. Please check.

Answer: OK

Query: Please confirm that given names and surnames have been identified correctly

Answer: Yes

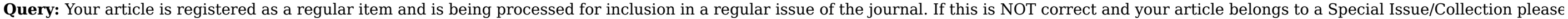
contact r.pauljames@elsevier.com immediately prior to returning your corrections.

Answer: Yes 